\title{
Announcement
}

\section{Journal of Pediatric Rehabilitation Medicine announces new Editor-in-Chief}

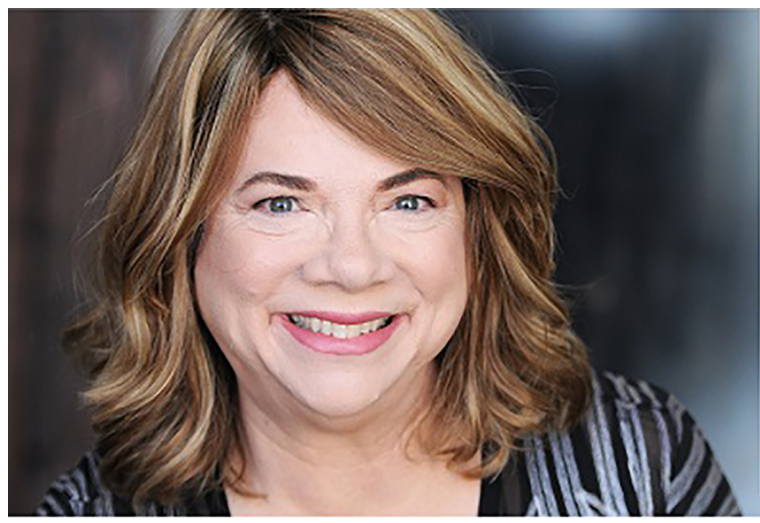

IOS Press is pleased to announce that Dr. Elaine Pico has been appointed as the new Editor-in-Chief of the journal. Dr. Pico is an attending physician in the Division of Pediatric Rehabilitation at UCSF Benioff Children's Hospital Oakland, California. She serves as co-principal investigator of NIH's Rett Syndrome MECP2 Duplication Disorder and Rett-Related Disorders Natural History Protocol. She holds a Master's degree in child life education, a doctorate in medicine from University of California in Los Angeles, and completed her residencies in pediatrics and physical medicine and rehabilitation at the Mayo Clinic. Dr. Pico is board certified by the American Board of Physical Medicine and Rehabilitation, American Board of
Pediatrics, and holds speciality certification in pediatric rehabilitation. She has published on such topics as the effect of low magnitude mechanical stimulation on bone density in patients with Rett syndrome. Dr. Pico continued Dr. Neufeld's research with the CDC's National Spina Bifida Registry (NSBR) at UCSF when he accepted the position as medical director in Boise, Idaho. She also participated in the Committee for Science and Publications where she reviewed and approved NSBR research manuscripts generated nationally. In 2014, Dr. Pico presented data from the CDC's National Spina Bifida Registry at the American Academy of Cerebral Palsy and Developmental Medicine's national meeting. Dr. Pico was lead author of the Spina Bifida chapter and contributing author for orthopedics and musculoskeletal conditions in both the 2010 and 2015 editions of Pediatric Rehabilitation Principles and Practice. She has lectured at Children's Hospital Oakland Research Institute and also internationally on mucopolysaccharidosis and cerebral palsy. In addition to research at UCSF, Dr. Pico has been working with Stanford University colleagues, students, and residents on various research projects for the past several years and enjoys teaching, imparting information, and publishing medical research.

Please join us in welcoming Dr. Elaine Pico! 